\title{
China Experience in Controlling COVID-19
}

\author{
Shiliang SONG \\ Affiliations: Chief physician and professor, Department of Internal Medicine, Taicang \\ Hospital affiliated to Suzhou University, Suzhou, China \\ Correspondence: zll5404@suda.edu.cn \\ Word count: 2789 . Character count 18314
}

\begin{abstract}
At present, the epidemic situation of COVID-19 is raging rampantly in the whole world, affecting the hearts of billions of people. In less than half a year, COVID19 swept the world, seriously threatening the safety of all mankind. At the beginning, the epidemic was most serious in China. Under the strong command of the highest level of the Chinese government, the whole Chinese people United as one, and achieved initial results in the struggle against covid-19 with scientific prevention and control. Summarizing China's experience and lessons in combating the epidemic is undoubtedly very beneficial to the people of the world in jointly combating the impact of the COVID-19 epidemic. The most important experience is: strong organizational leadership, enhanced cooperation with the WHO, the establishment of a national antiepidemic headquarters, responsible for the command and control of human, financial and material resources throughout the country, and local officials at all levels in the front line of the epidemic. Heart to heart with the people, breathe together and share a common destiny. Prevention is the first, prevention and treatment are combined. The treatment emphasizes the combination of Chinese and Western medicine. The "Four Anti and Two Balances" advocated by Academician Li Lanjuan's team, and the "Three Drugs and Three Formulas" recommended by Chinese medicine experts of the National and Health Commission, played a key role in improving the success rate of treatment. Establish fever clinics and establish square cabin hospital to eliminate infections in hospitals, emphasizing the protection of medical staff and avoiding crossinfection. Control the source of infection, try to achieve "four early", early detection, early isolation, early diagnosis and early treatment. "Four concentration", focus on patients, experts, resources and treatment. Take all measures to cut off the spread. Take all measures to protect susceptible people. Wearing masks, washing hands frequently, hot bathing, individual serving, home office, going out to maintain a social distance of more than one meter, eating nutritious foods rich in protein and vitamins, strengthening physical exercise and improving physical fitness are all effective ways to prevent COVID-19.
\end{abstract}

Keywords: COVID-19; COVID-19 prevention; COVID-19 treatment; Experience and lessons learned; prevention ; treatment ; China; Mobile square cabin hospital; mask; prevention of medical staff infection; Traditional Chinese medicine.

\section{Background and purpose}

At the beginning of the year, the COVID-19 epidemic that raged all over the world affected the hearts of hundreds of millions of people. Up to 8 May 2020 (the same as below, update the latest data when the last revision), the total number of confirmed patients in the world was 400 9911, of which 275934 died, ${ }^{[1]}$ with a mortality rate of 
$6.88 \%$ (this value is dynamic). 215 countries and regions were affected. ${ }^{[1]}$ In the past month, the world has seen an average of about one 80000 of new infections per day and about 5,800 deaths per day and continued. ${ }^{[1]}$ A total of 82886 patients were diagnosed and 4633 patients died in China, ${ }^{[1]}$ with a mortality rate of $5.59 \%$. At the beginning, the epidemic was most serious in China. Under the strong command of the highest level of the Chinese government, the whole Chinese people United as one, and achieved initial results in the struggle against covid-19 with scientific prevention and control. The number of new cases in China has been controlled below 10 per day. ${ }^{[2]}$ Summarizing China's experience and lessons in combating the epidemic is undoubtedly very beneficial to the people of the world in jointly combating the impact of the COVID-19 epidemic.

\section{Experience and lessons learned}

Organization and leadership: the state establishes an anti-epidemic headquarters, ${ }^{[2]}$ which is responsible for the general command and dispatch of human, financial and material resources throughout the country, and holds regular press conferences to keep the people of the whole country informed of the true situation of the epidemic. Local officials at all levels went deep into the front line of the epidemic, connected with the people's hearts, breathed with them and shared their destiny. The Ministry of Health of the People's Republic of China established a think tank of senior experts to be responsible for the revision of disease diagnosis and treatment plans and to update them in practice in a timely manner. "COVID-19 Diagnosis and Treatment Program (Trial)" has been updated from the first edition to the seventh edition. ${ }^{[3]}$ The diagnosis and treatment plan includes the etiology and epidemiological characteristics of COVID-19, pathological changes and clinical characteristics, diagnosis and differential diagnosis, clinical classification and early warning of critical illness, treatment principles and discharge standards, infection prevention and control in medical institutions, etc. ${ }^{[3]}$ It gives a detailed description in each aspect, and points out the direction for the diagnosis and treatment of medical workers across the country. It mobilized the nation's human, material, financial and medical resources to support the most severe epidemic in Wuhan, Hubei Province, and concentrated its superior forces to fight the epidemic.

Controlling the source of infection: The source of infection seen so far is mainly COVID-19 patients. ${ }^{[3]}$ People with asymptomatic infection may also become a source of infection. [3]"Four early", early detection, early isolation, early diagnosis and early treatment. [4] "Four concentration", focus on patients, experts, resources and treatment. ${ }^{[4]}$ Suspected cases were isolated and treated separately, with early detection of SARS-CoV-2 nucleic acid, and excluded homes were isolated for 14 days. After diagnosis, he was transferred to square cabin hospital for mildly and severely transferred to ICU. Square Cabin Hospital: Use stadiums, conference centers, convention centers and other large venues to transform into a temporary hospital that can accommodate hundreds of patients. ${ }^{[2]}$ The patients are admitted to mild patients who have been diagnosed as COVID-19 by nucleic acid detection. Its advantages are that it can be transformed in a short time of 2 to 3 days, it has a large capacity to accept patients, and there are medical staff for diagnosis and treatment and nursing and 
health education, while at home isolation, patients do not receive medical and nursing services, and are easily infected For family members and easy to cause community proliferation. On February 28, 2020, Ma Xiaowei, director of the National Health and Health Commission, said that Wuhan has built 16 square cabin hospitals, with more than 13,000 beds actually opened, accumulating more than 12,000 patients. $1 / 4$ patient was treated in the shelter hospital. The square cabin hospital has achieved zero infections, zero deaths, and zero turning points, and is truly a "cabin of life". ${ }^{[2]}$ Establish a fever clinic. Establish fever clinics in counties and districts. Suspected patients were given SARS-CoV-2 nucleic acid test. It was found that the patient was sent to a designated hospital for isolation treatment. Watch out for infections by medical staff. In the early stage of the disease, due to lack of knowledge about the disease, a large number of patients flowed into the hospital to cause medical runs, coupled with the shortage of protective materials, extreme fatigue of medical staff, psychological tension and other reasons, local medical staff in Hubei Province (mainly Wuhan City) infected COVID -19 more than 3000 people. ${ }^{[2,5]}$ However, since then, more than 42,000 national medical team members supporting Hubei Province (mainly Wuhan City) have taken measures such as strict personal protection, national medical supplies support, the combination of work and rest of medical staff, a balanced diet, adequate sleep, and intervention by psychologists. The implementation of the method has achieved zero infection, ${ }^{[2,5]} \mathrm{P}<0.001$, and the two are significantly different. Facts have proved that as long as strict isolation and disinfection are carried out and attention is paid to protection, SARS-COV- 2 can be prevented, and even close contact with medical staff can prevent cross-infection. All measures must be taken to control the initial areas of covid-19, such as searching for close contact, testing for SARS-COV-2, quarantine treatment for positive cases, close medical observation for 14 days for negative cases and second nucleic acid test the next day before the negative cases were released from medical observation. And the patients have been to the relevant places, such as airports, wharfs, shopping malls, hotels, residential areas and families and other places, strict disinfection. Put out the spark, not let it prairie fire, we must nip the epidemic in the bud! The treatment emphasizes the combination of Chinese and Western medicine. The "Four Anti and Two Balances" advocated by Academician Li Lanjuan 's team, and the "Three Drugs and Three Formulas" recommended by Chinese medicine experts of the National and Health Commission, played a key role in improving the success rate of treatment. Four Anti: anti-virus, anti-shock, anti-hypoxemia and multiple organ dysfunction, anti-secondary infection. Two Balance: maintain the acidbase balance of water electrolytes and maintain the micro-ecological balance. Three kinds of medicine: Jinhua Qinggan Granules, Lotus Qingwen Capsules, Xuebijing Injection. Three prescriptions: Qingfei Paidu Decoction, Huashi Baidu Recipe, Xuanfei Baidu Recipe. ${ }^{[3]}$

Cut off the transmission route: COVID-19 through the respiratory tract droplets close contact transmission is the main transmission route. Since SARS-CoV-2 can be isolated in feces and urine, it should be noted that feces and urine cause aerosol or contact transmission of environmental pollution. ${ }^{[3]}$ COVID-19 outbreak occurred during the Chinese New Year, and the flow of people was huge. Due to the timely closure of the 
city of Wuhan in Hubei Province, the outbreak of the outbreak was effectively blocked, which won time for prevention and control at home and abroad. According to different epidemic situations in different regions, a hierarchical control strategy is adopted. In high-risk areas with severe epidemics, the strategy of "internal non-proliferation, external prevention, and strict control" is implemented. ${ }^{[4]}$ In the medium-risk areas, implement the strategy of "importing external defense, preventing internal proliferation". Implement the strategy of "foreign defense import" in low-risk areas. ${ }^{[4]}$ The city takes community as the unit, and the township takes the villager group as the unit for isolation, closed management, personnel and vehicle entry and exit gate registration, and personnel temperature measurement. If you have fever and other discomforts, go to Fever Clinic for medical treatment Drive publicity vehicles, shuttle through the streets and alleys, and repeatedly broadcast the prevention and treatment of COVID-19 and the medical treatment process, so that they are well known. Stop the rally tour, delay the school start, stop the large banquet, stop the large-scale cultural sports and religious activities Close public places such as museums and large convention centers. Community and village committees, neighborhood committees, police, and community doctors carry out joint prevention and control.

Protection of susceptible groups: men, women and children, regardless of national skin color, the elderly and patients with underlying diseases are particularly susceptible to infection and have a high mortality rate after infection, and protection should be strengthened. Wear masks. During the COVID-19 epidemic, people across the country need to wear masks in public places, even in the vast rural areas on the edge, even if there are COVID-19 patients or asymptomatic carriers in the crowd. With the double mask protection between people, the probability of mutual infection will be greatly reduced. Everyone wears a mask, the prevention and treatment efficiency is the highest, and the cost is the lowest. Countries in the world must pay great attention to this. Another benefit of wearing a mask is that it avoids the bad habit of scratching the mouth and nose. Masks are based on N95 or surgical masks, but ordinary masks can also achieve effective protection, provided that all people wear masks. The poorer regional governments organize the supply of masks and distribute them to every household. Wash hands frequently, especially after contact with suspicious patients. You must wash your hands before and after meals, and rinse them with running water repeatedly. Reduce and eventually eliminate bad habits. Washing hands frequently, hot bathing, individual serving, home office, going out to maintain a social distance of more than one meter, eating nutritious foods rich in protein and vitamins, don't eat wild animals, strengthening physical exercise and improving physical fitness are all effective ways to prevent COVID-19.

\section{Discussion and summary}

China's successful experience in controlling COVID-19 is mainly based on the idea of a chess game in the nationwide, strong organizational leadership, and close cooperation with WHO. The country has established an anti-epidemic command headquarters, which is responsible for the overall command and dispatch of the country's human, financial and material resources. Local officials at all levels have penetrated the frontline of the epidemic and connected with the people, breathing together and 
sharing destiny. Carry out prevention first and combine prevention with treatment. The treatment emphasizes the combination of Chinese and Western medicine. The "Four Anti and Two Balances" advocated by Academician Li Lanjuan 's team, and the "Three Drugs and Three Formulas" recommended by Chinese medicine experts of the National and Health Commission, played a key role in improving the success rate of treatment. Take all measures to cut off the spread. Take all measures to protect susceptible people. Special emphasis is placed on three points: First, all measures must be taken to control COVID-19 initiating areas, follow close contacts, conduct SARS-CoV2 testing, positive patients should be isolated and treated, and negative patients must be closely observed for 14 days Strictly disinfect the relevant places visited by patients, such as airports, docks, shopping malls, hotels, residential quarters and families. Extinguishing the fire of the stars will not let it catch fire, and the epidemic must be wiped out in a budding state! This is of great significance to the prevention and control of the epidemic situation in the country where COVID-19 was first released and the region where it was first released in the same country. Second, wearing a mask is simple and convenient, and its prevention and treatment effectiveness is the first, especially medical personnel should be strictly in place. In Europe and the United States, the incidence rate is high, and the reluctance to wear a mask is the main reason. The painful lessons must be remembered and corrected. In the European and American countries, why the incidence rate is low, in addition to being taught by relatives and friends in the mainland, getting used to wearing mouth is the primary reason. Everyone wears a mask, the prevention and treatment efficiency is the highest, and the cost is the lowest. Countries in the world must pay great attention to this. Third, strengthen the protection of medical personnel. Medical personnel are the main force in the fight against COVID-19 and the main cause of cross-infection in hospitals, so they must be strictly protected and protected. It is necessary to provide qualified and sufficient protective materials, abide by strict disinfection and isolation regulations, ensure adequate rest time, maintain good physical and mental health, and create an atmosphere of respecting doctors and doctors.

As long as people all over the world unite as one, scientific decision-making, prevention is the first, prevention and treatment are combined, and we will continue to draw on and learn from the successful experience of all countries in the world, combine our national conditions, and use the wisdom of all mankind to deal with the common enemy SARS-CoV-2 and COVID- 19. We can definitely win this people's war against the epidemic. Dr. Tedros Director-General of WHO: "National unity and world unity. We are all united and will be able to defeat COVID-19". ${ }^{6]}$

\section{Acknowledgemen}

I thank Professor Xiaojian Yao, an international virologist, Professor Jin Jian, a member of China's COVID-19 Expert Group and Zhao Chao, chief physician, put forward some good suggestions for this article. Dr. Xu Ning contributed to the writing of this article, and my wife Huang fenglan, deputy director of the nurse to do a good job logistics support work. I want to thank all the experts who fought against COVID-19 and the Chinese doctors and nurses, your hard work has successfully prevented the popularity 
of COVID-19 in China.

\section{References}

[1] World meter. https://www.worldometers.info/coronavirus/\#countries

[2] The official website of National Health Commission of the People's Republic of China, www.nhc.gov.cn.

[3] The General Office of the National Health Council of China and the Office of the Administration of Traditional Chinese Medicine. Diagnosis and treatment of COVID 19 (trial version 7), March 3, 2020, www.chinanews.com

[4] The General Office of the National Health Council of China COVID-19 programme for provention and control. (trial version 6) www.nhc.gov.cn.

[5]The Novel Coronavirus Pneumonia Emergency Response Epidemiology Team. The Epidemiological Characteristics of an Outbreak of 2019 Novel Coronavirus Diseases (COVID-19) in China, 2020[J]. China CDC Weekly, 2020, 2(8): 113-122.

doi: $10.46234 /$ ccdcw2020.032

[6]Speech by the Director-General of WHO at the media briefing of the 2019 Coronavirus Disease (COVID-19) outbreak on May 6, 2020, www.who.int 